\title{
Impact of Global Economic Crisis on Export Diversification of Agricultural Commodities: The Case of Indian Horticulture Market
}

\author{
Dr. Ali Ghufran ${ }^{1}$, Syed Fahad Ashraf ${ }^{2}$ \\ I'Assistant Professor, Department of Business Management, Integral University, India) \\ ${ }_{2}^{2}$ (Research Scholar, Department of Business Management, Integral University, India)
}

\begin{abstract}
The present external environment is conducive to export expansion and emerge India as an important exporter for agricultural commodities, but the post crisis slump in the world commodity market and declining commodity prices, forced India to diversify export of its agricultural commodities from traditional to non traditional exports to recover from the slowdown. The economic recession of September 2008 which first emerged as a financial crisis in one country has now spread his hand in almost all corners of the globe but the developing economy like India which is still in slow down stage and not yet in recession, is struggling tough to vanish this. The present paper examines some of the striking trends of India's exports of overall agricultural commodities in general and horticultural commodities in particular during the last decade. The Herfindahl index has been used to analyze the export diversification in agricultural commodities. As densification is one of the effective measures to mitigate the risk during crisis, this study provides practical insights for exporters and policy makers for designing effective framework to promote export during risky period.
\end{abstract}

Keywords: - CAGR, Densification, Export diversification, Gross Domestic Product, Herfindahl Index

\section{Introduction}

The global financial crisis of late 2000 has caused the emerging and developing economies to replace advanced economies to lead global economic growth. Advanced economies accounted for only $35 \%$ of incremental global nominal GDP and 23\% of incremental global GDP (PPP) while emerging and developing economies accounted for $65 \%$ of incremental global nominal GDP and $77 \%$ of incremental global GDP (PPP) from 2007 - 2011 according to International Monetary Fund (IMF). As per IMF statistics of 2007-2011, India placed $5^{\text {th }}$ position for incremental nominal GDP, while for incremental nominal GDP (PPP) its rank was $2^{\text {nd }}$. Before economic recession of 2008, in the year 2007, world trade in fruits was worth USD 60.6 billion, and that of vegetables was worth USD 44.7 billion, growing at a CAGR of $12.36 \%$ and $12.68 \%$ respectively while India's share in world exports of fruits and vegetables are less than $1.5 \%$, India's exports of fruits and vegetables have grown at a CAGR of $20.61 \%$ and $7.21 \%$ respectively by value between 2004 and 2007. Export growth in India has been much faster than GDP growth over the past few decades and demand for Indian exports increases when its export prices fall in relation to world prices. Export supply is positively related to the domestic relative price of exports and higher domestic demand reduces export supply. The global economical crisis resulted in the collapse of large financial institution but its impact affects and spread to all the economies, and India too cannot escape from it, which affect its export activities as well and force India to diversify its export activities to lead higher growth to help them to overcome export instability or the negative impact of terms of trade.

Agricultural diversification has no unique meaning but it is a process whereby more and more activities are added either to a single farm enterprise or to a region. Although literature on agricultural export diversification and economic growth has been quite rich in analytical insights, relatively little empirical work of a statistical nature has been undertaken on the subject. Agricultural diversification could be pursued with two broad objectives in mind: First, to expand the production and distribution of agricultural commodities to satisfy a higher proportion of domestic food consumption where agricultural diversification is seen as a means of generating domestic food security, or as an import substitution policy. Secondly, to increase the mix of agricultural commodities targeted for the export market in order to stabilize foreign exchange earnings.

\section{Objectives of the Study}

The objective of the present study is: -

1) To study the performance of growth and stability of volumes (produced and exported) and value of agricultural commodities. 
2) To study the percentage distribution for horticultural commodities to that of total export of the agricultural commodities.

3) To find out the concentration ratio with the help of Herfindahl Index in order to study competition and market power of agricultural commodities in general and horticulture commodities in particular.

\section{Research Methodology}

To determine the above objectives, secondary data has been collected for total agricultural commodities exported from India for the last 10 years (2001-2010) in order to find out the diversification or concentration based on the value of agricultural commodities in general and horticultural commodities in particular.

\section{Data Sources}

The secondary data has been collected through the following sources: -

(a) Monthly Statistics of Foreign Trade, Ministry of Commerce

(b) Agricultural Produce Export Development Authority (APEDA)

(c) Internet

\section{Data Analysis}

Export trends in agricultural commodities are examined over the period 2001-2010 in order to find out the diversification / concentration with the help of Herfindahl Index which is an indicator of the amount of competition. The Herfindahl index equals the sum of the squared proportional export to each commodity group within agricultural produce and hence has a maximum of 1 when the country is completely focusing on one commodity group, so lower values of the index indicate more diversification. Increase in the HI generally indicates a decrease in competition and an increase of market power, where as decreases indicates the opposite. One can calculate Herfindahl index as -

$$
H=\sum_{i=1}^{N} s_{i}^{2}
$$

where $s_{i}$ is the market share of commodity $i$ in the market, and $N$ is the total number of commodities.

- A HI index below 0.01 indicates a highly competitive index.

- A HI index below 0.15 indicates an un-concentrated index.

- A HI index between $0.15-0.25$ indicates moderate concentration.

- A HI index above 0.25 indicates high concentration.

\section{Interpretation}

From annexure 1, 2 and 3, the researcher comes to a conclusion that the $\mathrm{HI}$ for total agricultural exports during 2001-02 to 2009-10 lies between 0.060 and 0.079 , which clearly indicates that there was an increase in competition for export of agricultural commodities. Similarly the HI for fruits and vegetables for the same period which lies between 0.018 to 0.042 and 0.110 to 0.417 respectively speaks itself that the country is not focussing on one commodity group and these small index clearly indicates a competitive industry with dominant commodities..

\section{Changes in Agricultural Exports}

By compiling the 10 year data (Annexure 1) as shown in Fig. 1, it is found that the export of overall agricultural commodities, which was 11641.12 MT in the year 2001-02, increase to 15279.20 MT in 2005-06 and slightly decrease to 14623.86 MT in 2009-10. Contrary to this the value of total exported agricultural commodities, which was Rs. 29728.61 Crore in the year 2001-02 rose to Rs. 49217.00 Crore in 2005-06 and further increase to Rs. 89522.59 in 2009-10.

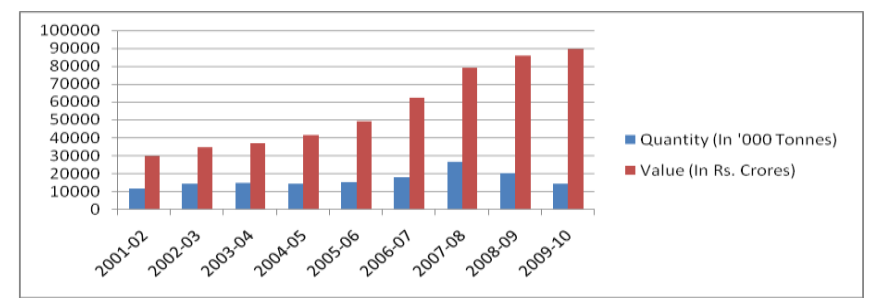

Source: - Calculation Based on data of Monthly Statistics of Foreign Trade, Ministry of Commerce, GOI

Figure 1: Quantity / Value of Agricultural Commodities exported during 2001-02 to 2009-10 
This clearly indicates that despite slump in world market due to recession of 2008, Indian agricultural commodities succeed to achieve a high export growth rate between 2008-09 to 2009-2010. In order to find out the growth rate during the first and last financial year of the previous decade, the researcher calculates the Compound Annual Growth Rate (CAGR) of 11 groups of agricultural commodities as shown in Table 1, which depicts that the growth rate of all the commodities lies between $6 \%$ to $19 \%$, excluding sugar whose growth rate was negative. While the CAGR for fruits and vegetables were just $15 \%-16 \%$.

Table 1: Trends in Agricultural Exports, 2001-02 to 2009-10 (Value in Rs. Crores)

\begin{tabular}{|l|c|c|c|}
\hline \multicolumn{1}{|c|}{ Commodities } & $\mathbf{2 0 0 1 - 0 2}$ & $\mathbf{2 0 0 9 - 1 0}$ & CAGR (\%) 2001-10 \\
\hline Food grains & 4989.40 & 14665.91 & 12.72 \\
\hline Tea and Coffee & 2814.14 & 4976.27 & 6.55 \\
\hline Fruits & 929.70 & 3424.90 & 15.58 \\
\hline Vegetables & 1560.36 & 5938.63 & 16.02 \\
\hline Animal Products & 1549.53 & 7200.58 & 8.48 \\
\hline Nuts & 2044.87 & 4254.15 & -25.23 \\
\hline Sugar & 1781.85 & 130.00 & 9.75 \\
\hline Floriculture & 127.43 & 293.98 & 17.04 \\
\hline Spices & 1496.97 & 6161.02 & 13.99 \\
\hline Oilseeds & 3902.04 & 12679.62 & 14.90 \\
\hline Other Products & 8532.32 & 29797.53 & \\
\hline
\end{tabular}

Source: - Calculation Based on data of Monthly Statistics of Foreign Trade, Ministry of Commerce, GOI

\section{Structure of Indian Horticultural Export Market}

India is a large, low-cost producer of fruit and vegetables, and horticulture is a sector with huge export potential. But, high transportation costs, inadequate storage facilities, a fragmented supply chain, and weak quality standards at home are eroding its competitiveness. Rising incomes and growing consumer interest in a variety of fresh fruits and vegetables year-round is stimulating international trade in horticulture. India being a home of wide variety of fruits and vegetables holds a unique position in production figures among other countries and is one of the world's biggest producers of horticultural products growing nearly 11 percent of all the world's vegetables and 15 percent of all fruit, and its production costs are less than half of those in other parts of the world. Despite these advantages, India's share in the global market is insignificant - it accounts for only 1.7 percent of the global trade in vegetables and 0.5 percent in fruits. India's own share of fruits and vegetables export to that of its total agricultural exports rose from 3.34\% (2001-02) to 12.01 (2009-10) and 2.60 (2001-02) to 12.30 (2009-10), but still not enough to cater need of world commodity market despite being the world's biggest producer of horticultural produce (Fig. 2).

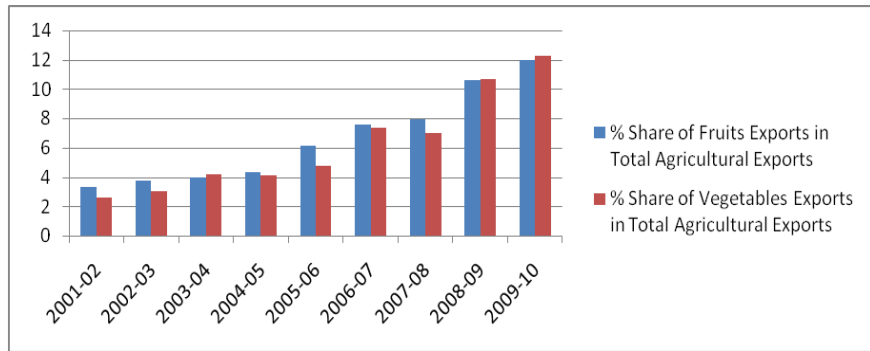

Source: - Calculation Based on data of Monthly Statistics of Foreign Trade, Ministry of Commerce, GOI Figure 2: Percentage Share of Fruits \& Vegetables Export to that of Total Agricultural Exports 


\section{Diversification in Agricultural / Horticultural Export}

Fig. 3 clearly shows that, for the export of total agricultural products, the value of HI from 2001-02 to 2009-10 lies between $(0.60<\mathrm{HI}<0.79)$ and clearly indicates high concentration as the value is above 0.25 . Similarly from Annexure 2 and 3, the HI value for selected fruits $(0.016<\mathrm{HI}<0.042)$ shows an un-concentrated index from 2001-02 to 2009-10 being value $\mathrm{HI}<0.15$, while for selected vegetables $(0.08<\mathrm{HI}<0.26)$ shows an un-concentrated index as well as moderate concentration from 2001-02 to 2006-2007, and high concentration between 2007-08 to 2009-10 as the value of HI during these three years was above 0.25.

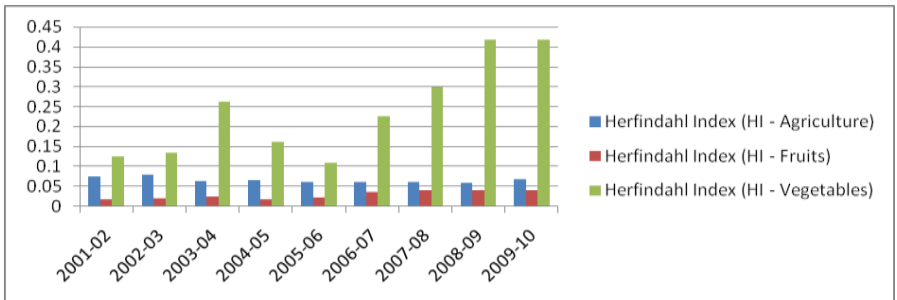

Source: - Calculation Based on data of Monthly Statistics of Foreign Trade, Ministry of Commerce, GOI Figure 3: Diversification in Agricultural Exports, 2001-02 to 2009-10 (HI)

As per Table 2, the percentage share related to diversification of fruits and vegetables to that of total agricultural exports was a meager figure being just only between $3 \%$ to $6 \%$ for the period 2001-2010. It shows a growth rate of approx 3\% - 4\% for fruits while the growth rate for vegetables was between 5\% - 7\%, to that of total agricultural exports.

Table 2: Diversification in Agricultural Exports, 2001-02 to 2009-10 (Share in \%)

\begin{tabular}{|l|c|c|c|}
\hline \multicolumn{1}{|c|}{ Commodities } & $\mathbf{2 0 0 1 - 0 2}$ & $\mathbf{2 0 0 5 - 0 6}$ & $\mathbf{2 0 0 9 - 1 0}$ \\
\hline Food grains & 16.78 & 16.95 & 16.38 \\
\hline Tea and Coffee & 9.47 & 6.74 & 5.56 \\
\hline Fruits & 3.13 & 3.50 & 3.83 \\
\hline Vegetables & 5.25 & 5.06 & 6.63 \\
\hline Animal Products & 5.21 & 7.83 & 8.04 \\
\hline Nuts & 6.88 & 6.30 & 4.75 \\
\hline Sugar & 5.99 & 1.21 & 0.16 \\
\hline Floriculture & 0.42 & 0.60 & 0.33 \\
\hline Spices & 5.04 & 4.29 & 6.88 \\
\hline Oilseeds & 13.13 & 15.67 & 14.16 \\
\hline Other Products & 28.70 & 31.85 & 33.28 \\
\hline HI (Fruits) & 0.019 & 0.023 & 0.042 \\
\hline HI (Vegetables) & 0.125 & 0.110 & 0.417 \\
\hline HI (Agriculture) & 0.075 & 0.062 & 0.069 \\
\hline
\end{tabular}

Source: - Calculation Based on data of Monthly Statistics of Foreign Trade, Ministry of Commerce, GOI

By summing up, the researcher came to the conclusion that the value of HI, which was 0.075 in the year 2001-02 increased to 0.079 in the year 2002-03 but decreased to 0.063 in the year 2003-04 which further increased to 0.066 in the year 2004-05. The data again showing decreasing trend till 2008-09 but increased in the year 2009-10, which clearly indicates that during the period 2001-2010, India's export of agricultural commodities was totally diversified as during these periods the value of $\mathrm{HI}$ is less than 1 . Similarly, calculated HI for selected 11 fruits and vegetables from year 2001-2010, the researcher get the value of HI $(0.019<\mathrm{HI}<0.042)$ for fruits and $(0.110<\mathrm{HI}<0.417)$ for vegetables, which also indicates that during these periods there was heavy diversification in the export of horticultural products.

\section{Conclusion and Policy Implications}

The novelty of this paper is to use detail information related to export of agricultural commodities in general and horticulture commodities in particular. In addition, using these information's the researcher calculates Herfindahl Index in order to know the export diversification in agricultural commodities. Following the objectives set out at the start of the paper, the main findings of the paper are as follows: -

- Value of agricultural commodities exported during the last three years has been increased from Rs. 79039.72 Crore (2007-08) to Rs. 89522.59 (2009-10) while the volume has been decreased from 26809.80 in '000 MT (2007-08) to 14623.86 in '000 MT (2009-10). 
- From 2001-02 to 2009-10, the CAGR for fruits and vegetables are much higher than the CAGR of total agricultural exports during the same period.

- Percentage share of fruits increased more than thrice, while of vegetables increased approx 5 times to that of total agricultural exports for the last decade, and finally

- The HI value for the last decade clearly indicates that there was diversification in the export of agricultural and horticultural commodities, which offers an attractive option and a major source of pushing up growth of agricultural sector. The price competitiveness of India's horticultural commodities, like that of any other traditional commodities has determined their export performance over time. While technological upgradation and associated institutional changes are identified as thrust areas for future development of the horticulture sector, exports are considered to be most important for the growth of the sector. India can retain its price competitiveness in horticultural commodities with a depreciating currency in addition to India's low cost conditions can look forward to emerge as a major producer of horticultural commodities and thus secure reasonable market access for its agro exports, which are largely dependent on the competitive technologies that will help in enhancing export potential. This development will also help in overall growth of the economy through generation of extra foreign exchange, creating employment opportunities and also upliftment of the small and marginal farmers.

The window of international demand for the horticultural products is very small thus a planned core marketing strategies has to be implemented to target the markets which will help in future expansion of the domestic and international markets by overcoming export barriers.

\section{References}

[1]. Data on India's Export of Agricultural Products (2001-02 to 2009-10), Official website of Ministry of Commerce, Government of India, URL: http://commerce.nic.in/eidb/default.asp

Annexure: 1

India's Export of Agricultural Products

(Quantity: In '000 Tonnes, Value: In Rs. Crores)

\begin{tabular}{|c|c|c|c|c|c|c|c|c|c|}
\hline \multirow{2}{*}{ Commodity } & \multicolumn{3}{|c|}{ 2001-02 } & \multicolumn{3}{|c|}{$2002-03$} & \multicolumn{3}{|c|}{$2003-04$} \\
\hline & $\begin{array}{r}\text { Quantit } \\
\mathbf{y} \\
\end{array}$ & Value & $\begin{array}{r}\text { HI } \\
(\mathrm{Ag}) \\
\end{array}$ & $\begin{array}{r}\text { Quantit } \\
\mathbf{y} \\
\end{array}$ & Value & $\begin{array}{r}\text { HI } \\
(\mathrm{Ag}) \\
\end{array}$ & $\begin{array}{r}\text { Quantit } \\
\mathbf{y} \\
\end{array}$ & Value & $\begin{array}{r}\text { HI } \\
(\mathrm{Ag}) \\
\end{array}$ \\
\hline Pulses & 161.64 & 369.13 & 0.000 & 148.08 & 345.02 & $\begin{array}{r}0.00 \\
0 \\
\end{array}$ & 153.88 & 328.60 & $\begin{array}{r}0.00 \\
0 \\
\end{array}$ \\
\hline Rice Basmati & 667.07 & 1842.77 & 0.004 & 708.79 & 2058.47 & $\begin{array}{r}0.00 \\
4 \\
\end{array}$ & 771.49 & 1993.05 & $\begin{array}{r}0.00 \\
3 \\
\end{array}$ \\
\hline $\begin{array}{l}\text { Rice(Other } \\
\text { than Basmati) }\end{array}$ & 1541.49 & 1331.37 & $\begin{array}{r}0 .) 00 \\
2 \\
\end{array}$ & 4259.08 & 3772.77 & $\begin{array}{r}0.01 \\
2 \\
\end{array}$ & 2640.57 & 2174.94 & $\begin{array}{r}0.00 \\
3 \\
\end{array}$ \\
\hline Wheat & 2649.38 & 1330.21 & 0.002 & 3671.25 & 1759.87 & $\begin{array}{r}0.00 \\
3\end{array}$ & 4093.08 & 2391.15 & $\begin{array}{r}0.00 \\
4\end{array}$ \\
\hline Other Cereals & 144.73 & 115.92 & 0.000 & 106.08 & 91.06 & $\begin{array}{r}0.00 \\
0 \\
\end{array}$ & 604.23 & 397.55 & $\begin{array}{r}0.00 \\
0 \\
\end{array}$ \\
\hline Tea & 180.10 & 1719.22 & 0.003 & 182.86 & 1652.07 & $\begin{array}{r}0.00 \\
2 \\
\end{array}$ & 177.77 & 1637.35 & $\begin{array}{r}0.00 \\
2 \\
\end{array}$ \\
\hline Coffee & 176.26 & 1094.92 & 0.001 & 184.87 & 993.98 & $\begin{array}{r}0.00 \\
1\end{array}$ & 188.44 & 1085.92 & $\begin{array}{r}0.00 \\
1\end{array}$ \\
\hline $\begin{array}{l}\text { Tobacco } \\
\text { Unmanufactur } \\
\text { ed }\end{array}$ & 84.48 & 582.05 & 0.000 & 100.47 & 733.52 & $\begin{array}{r}0.00 \\
0 \\
\end{array}$ & 120.64 & 801.41 & $\begin{array}{r}0.00 \\
0\end{array}$ \\
\hline $\begin{array}{l}\text { Tobacco } \\
\text { Manufactured }\end{array}$ & 13.46 & 225.66 & 0.000 & & 289.37 & $\begin{array}{r}0.00 \\
0 \\
\end{array}$ & & 295.06 & $\begin{array}{r}0.00 \\
0\end{array}$ \\
\hline $\begin{array}{l}\text { Poultry \& } \\
\text { Dairy Products }\end{array}$ & & 207.96 & 0.000 & & 176.45 & $\begin{array}{r}0.00 \\
0 \\
\end{array}$ & & 161.56 & $\begin{array}{r}0.00 \\
0 \\
\end{array}$ \\
\hline $\begin{array}{l}\text { Floriculture } \\
\text { Products }\end{array}$ & & 127.43 & 0.000 & & 180.77 & $\begin{array}{r}0.00 \\
0 \\
\end{array}$ & & 250.47 & $\begin{array}{r}0.00 \\
0 \\
\end{array}$ \\
\hline Spices & 239.29 & 1496.97 & 0.003 & 277.02 & 1655.49 & $\begin{array}{r}0.00 \\
2 \\
\end{array}$ & 267.47 & 1544.18 & $\begin{array}{r}0.00 \\
2 \\
\end{array}$ \\
\hline $\begin{array}{l}\text { Cashewnut } \\
\text { Shell Liquid }\end{array}$ & 1.99 & 5.25 & 0.000 & 6.14 & 8.56 & $\begin{array}{r}0.00 \\
0\end{array}$ & 4.33 & 5.02 & $\begin{array}{r}0.00 \\
0\end{array}$ \\
\hline
\end{tabular}


Impact Of Global Economic Crisis On Export Diversification Of Agricultural Commodities: The Case Of Indian Horticulture Market

\begin{tabular}{|c|c|c|c|c|c|c|c|c|c|}
\hline Cashew & 98.20 & 1788.68 & 0.004 & 129.43 & 2052.94 & $\begin{array}{r}0.00 \\
4\end{array}$ & 99.68 & 1699.82 & $\begin{array}{r}0.00 \\
2\end{array}$ \\
\hline Sesamum Seed & 218.97 & 562.23 & 0.000 & 118.31 & 372.89 & $\begin{array}{r}0.00 \\
0\end{array}$ & 189.11 & 708.90 & $\begin{array}{r}0.00 \\
0\end{array}$ \\
\hline Nigerseed & 22.22 & 47.85 & 0.000 & 36.13 & 77.99 & $\begin{array}{r}0.00 \\
0\end{array}$ & 17.89 & 45.41 & $\begin{array}{r}0.00 \\
0 \\
\end{array}$ \\
\hline Groundnut & 112.81 & 250.94 & 0.000 & 67.89 & 178.30 & $\begin{array}{r}0.00 \\
0 \\
\end{array}$ & 176.93 & 179.11 & $\begin{array}{r}0.00 \\
0 \\
\end{array}$ \\
\hline Guargum Meal & 117.89 & 403.09 & 0.000 & 111.94 & 486.64 & $\begin{array}{r}0.00 \\
0\end{array}$ & 119.33 & 120.56 & $\begin{array}{r}0.00 \\
0\end{array}$ \\
\hline Oil Meals & 2781.72 & 2262.93 & 0.006 & 1776.13 & 1487.35 & $\begin{array}{r}0.00 \\
2\end{array}$ & 3172.31 & 3249.89 & $\begin{array}{r}0.00 \\
8\end{array}$ \\
\hline Castor Oil & 213.68 & 625.94 & 0.000 & 177.69 & 609.81 & $\begin{array}{r}0.00 \\
0\end{array}$ & 152.36 & 656.06 & $\begin{array}{r}0.00 \\
0\end{array}$ \\
\hline Shellac & 5.70 & 72.99 & 0.000 & 5.72 & 89.85 & $\begin{array}{r}0.00 \\
0\end{array}$ & 10.50 & 179.74 & $\begin{array}{r}0.00 \\
0\end{array}$ \\
\hline Sugar & 1456.45 & 1728.29 & 0.003 & 1662.37 & 1769.49 & $\begin{array}{r}0.00 \\
3 \\
\end{array}$ & 1200.60 & 1216.59 & $\begin{array}{r}0.00 \\
1\end{array}$ \\
\hline Molasses & 221.12 & 53.56 & 0.000 & 207.86 & 45.05 & $\begin{array}{r}0.00 \\
0\end{array}$ & 98.62 & 19.38 & $\begin{array}{r}0.00 \\
0\end{array}$ \\
\hline $\begin{array}{l}\text { Fruits/Vegetab } \\
\text { le Seeds }\end{array}$ & 4.93 & 62.19 & 0.000 & 8.92 & 97.96 & $\begin{array}{r}0.00 \\
0\end{array}$ & 5.18 & 53.61 & $\begin{array}{r}0.00 \\
0\end{array}$ \\
\hline Fresh Fruits & & 417.15 & 0.000 & & 447.32 & $\begin{array}{r}0.00 \\
0\end{array}$ & & 784.03 & $\begin{array}{r}0.00 \\
0\end{array}$ \\
\hline $\begin{array}{l}\text { Fresh } \\
\text { Vegetables }\end{array}$ & & 575.20 & 0.000 & & 642.78 & $\begin{array}{r}0.00 \\
0\end{array}$ & & 953.93 & $\begin{array}{r}0.00 \\
1\end{array}$ \\
\hline $\begin{array}{l}\text { Processed } \\
\text { Vegetables }\end{array}$ & & 198.79 & 0.000 & & 256.73 & $\begin{array}{r}0.00 \\
0\end{array}$ & & 291.15 & $\begin{array}{r}0.00 \\
0\end{array}$ \\
\hline $\begin{array}{l}\text { Processed } \\
\text { Fruit Juices }\end{array}$ & & 512.55 & 0.000 & & 574.13 & $\begin{array}{r}0.00 \\
0\end{array}$ & & 343.66 & $\begin{array}{r}0.00 \\
0\end{array}$ \\
\hline $\begin{array}{l}\text { Miscellaneous } \\
\text { Processed } \\
\text { Items }\end{array}$ & & 724.18 & 0.001 & & 910.08 & $\begin{array}{r}0.00 \\
1 \\
\end{array}$ & & 1058.69 & $\begin{array}{r}0.00 \\
1 \\
\end{array}$ \\
\hline $\begin{array}{l}\text { Meat \& } \\
\text { Preparations }\end{array}$ & & 1193.28 & 0.002 & & 1377.19 & $\begin{array}{r}0.00 \\
2 \\
\end{array}$ & & 1714.41 & $\begin{array}{r}0.00 \\
2\end{array}$ \\
\hline $\begin{array}{l}\text { Marine } \\
\text { Products }\end{array}$ & 468.03 & 5898.34 & 0.039 & 527.87 & 6928.05 & $\begin{array}{r}0.04 \\
0\end{array}$ & 409.49 & 6105.63 & $\begin{array}{r}0.02 \\
7\end{array}$ \\
\hline $\begin{array}{l}\text { Cotton Raw } \\
\text { including } \\
\text { Waste }\end{array}$ & 8.23 & 42.69 & 0.000 & 11.75 & 50.28 & $\begin{array}{r}0.00 \\
0\end{array}$ & 179.61 & 942.37 & $\begin{array}{r}0.00 \\
1\end{array}$ \\
\hline Jute Hessian & 51.28 & 186.22 & 0.000 & & 349.31 & $\begin{array}{r}0.00 \\
0\end{array}$ & & 410.11 & $\begin{array}{r}0.00 \\
0\end{array}$ \\
\hline $\begin{array}{l}\text { Poultry } \\
\text { Products }\end{array}$ & & 148.29 & 0.000 & & 182.07 & $\begin{array}{r}0.00 \\
0\end{array}$ & & 253.59 & $\begin{array}{r}0.00 \\
0\end{array}$ \\
\hline $\begin{array}{l}\text { Paper/Wood } \\
\text { products }\end{array}$ & & 1524.37 & 0.003 & & 1950.33 & $\begin{array}{r}0.00 \\
3\end{array}$ & & 2362.58 & $\begin{array}{r}0.00 \\
4\end{array}$ \\
\hline $\begin{array}{l}\text { Total } \\
\text { Agricultural } \\
\text { Exports }\end{array}$ & & 29728.61 & 1.000 & & 34653.94 & $\begin{array}{r}1.00 \\
0\end{array}$ & & 37266.52 & $\begin{array}{r}1.00 \\
0\end{array}$ \\
\hline $\begin{array}{l}\text { Total National } \\
\text { Exports }\end{array}$ & & $\begin{array}{r}209017.9 \\
7\end{array}$ & & & $\begin{array}{r}255137.2 \\
8\end{array}$ & & & $\begin{array}{r}293366.7 \\
5\end{array}$ & \\
\hline \multirow{3}{*}{$\begin{array}{l}\text { \% Share of } \\
\text { Agricultural } \\
\text { Exports in } \\
\text { National } \\
\text { Exports }\end{array}$} & & 14.22 & & & 13.58 & & & 12.70 & \\
\hline & & & & & & & & & \\
\hline & & & 0.075 & & & $\begin{array}{r}0.07 \\
9\end{array}$ & & & $\begin{array}{r}0.06 \\
3\end{array}$ \\
\hline
\end{tabular}


Impact Of Global Economic Crisis On Export Diversification Of Agricultural Commodities: The Case Of Indian Horticulture Market

\begin{tabular}{|c|c|c|c|c|c|c|c|c|c|}
\hline \multirow{2}{*}{ Commodity } & \multicolumn{3}{|c|}{$2004-2005$} & \multicolumn{3}{|c|}{$2005-2006$} & \multicolumn{3}{|c|}{$2006-2007$} \\
\hline & Quantity & Value & $\begin{array}{r}\mathrm{HI} \\
(\mathrm{Ag})\end{array}$ & Quantity & Value & $\begin{array}{r}\mathrm{HI} \\
(\mathrm{Ag})\end{array}$ & Quantity & Value & $\begin{array}{r}\mathrm{HI} \\
(\mathrm{Ag})\end{array}$ \\
\hline Pulses & 271.18 & 602.57 & 0.000 & 447.44 & 1115.21 & 0.001 & 250.7 & 773.34 & 0.000 \\
\hline Rice Basmati & 1163 & 2823.9 & 0.005 & 1166.57 & 3043.1 & 0.004 & 1045.73 & 2792.81 & 0.002 \\
\hline $\begin{array}{l}\text { Rice(Other than } \\
\text { Basmati) }\end{array}$ & 3615.1 & 3945.02 & 0.009 & 2921.6 & 3178.17 & 0.004 & 3702.22 & 4243.10 & 0.005 \\
\hline Wheat & 2009.35 & 1459.82 & 0.001 & 746.18 & 557.53 & 0.000 & 46.64 & 35.35 & 0.000 \\
\hline Other Cereals & 1178.03 & 793.82 & 0.000 & 567.22 & 453.82 & 0.000 & 730.38 & 599.25 & 0.000 \\
\hline Tea & 183.4 & 1840.3 & 0.002 & 162.86 & 1730.73 & 0.001 & 185.63 & 1969.51 & 0.001 \\
\hline Coffee & 167.55 & 1069.08 & 0.001 & 177.68 & 1588.69 & 0.001 & 213.65 & 1969.00 & 0.001 \\
\hline $\begin{array}{l}\text { Tobacco } \\
\text { Unmanufactured }\end{array}$ & 135.74 & 940.07 & 0.001 & 142.7 & 1021.32 & 0.000 & 158.25 & 1251.28 & 0.000 \\
\hline $\begin{array}{l}\text { Tobacco } \\
\text { Manufactured }\end{array}$ & & 314.54 & 0.000 & & 309.34 & 0.000 & & 433.89 & 0.000 \\
\hline $\begin{array}{l}\text { Poultry \& Dairy } \\
\text { Products }\end{array}$ & & 458.79 & 0.000 & & 794.61 & 0.000 & & 497.09 & 0.000 \\
\hline $\begin{array}{l}\text { Floriculture } \\
\text { Products }\end{array}$ & & 222.92 & 0.000 & & 301.45 & 0.000 & & 652.70 & 0.000 \\
\hline Spices & 364.53 & 1883.18 & 0.002 & 400.24 & 2115.98 & 0.002 & 482.8 & 3157.90 & 0.003 \\
\hline $\begin{array}{l}\text { Cashewnut Shell } \\
\text { Liquid }\end{array}$ & 5.33 & 11.94 & 0.000 & 5.94 & 8.67 & 0.000 & 8.09 & 15.34 & 0.000 \\
\hline Cashew & 118.11 & 2477.18 & 0.004 & 125.1 & 2584.7 & 0.003 & 122.78 & 2291.18 & 0.001 \\
\hline Sesamum Seed & 168.28 & 708.95 & 0.000 & 199.81 & 746.6 & 0.000 & 233.34 & 939.58 & 0.000 \\
\hline Nigerseed & 26.14 & 64.74 & 0.000 & 28.42 & 60.25 & 0.000 & 30.02 & 66.87 & 0.000 \\
\hline Groundnut & 177.15 & 547.02 & 0.000 & 190.06 & 513.69 & 0.000 & 251.43 & 798.46 & 0.000 \\
\hline Guargum Meal & 131.31 & 689.48 & 0.000 & 186.73 & 1094.23 & 0.000 & 189.33 & 1125.79 & 0.000 \\
\hline Oil Meals & 3603.38 & 3177.6 & 0.006 & 5976 & 4875.01 & 0.010 & 6437.43 & 5504.32 & 0.008 \\
\hline Castor Oil & 271.69 & 1077.98 & 0.001 & 254.72 & 939.74 & 0.000 & 294.87 & 1090.11 & 0.000 \\
\hline Shellac & 8.54 & 164.87 & 0.000 & 9.3 & 159.98 & 0.000 & 7.51 & 147.20 & 0.000 \\
\hline Sugar & 108.69 & 149.53 & 0.000 & 321.2 & 569.1 & 0.000 & 1643.4 & 3127.47 & 0.003 \\
\hline Molasses & 8.16 & 5.52 & 0.000 & 72.94 & 28.81 & 0.000 & 326.87 & 133.37 & 0.000 \\
\hline $\begin{array}{l}\text { Fruits/Vegetable } \\
\text { Seeds }\end{array}$ & 6.74 & 66.04 & 0.000 & 7.52 & 92.96 & 0.000 & 8.1 & 121.59 & 0.000 \\
\hline Fresh Fruits & & 862.26 & 0.000 & & 1120.69 & 0.001 & & 1413.98 & 0.001 \\
\hline Fresh Vegetables & & 862.99 & 0.000 & & 919.81 & 0.000 & & 1546.53 & 0.001 \\
\hline $\begin{array}{l}\text { Processed } \\
\text { Vegetables }\end{array}$ & & 362.46 & 0.000 & & 494.48 & 0.000 & & 650.23 & 0.000 \\
\hline $\begin{array}{l}\text { Processed Fruit } \\
\text { Juices }\end{array}$ & & 369.16 & 0.000 & & 599.91 & 0.000 & & 711.40 & 0.000 \\
\hline $\begin{array}{l}\text { Miscellaneous } \\
\text { Processed Items }\end{array}$ & & 908.03 & 0.000 & & 989.53 & 0.000 & & 1125.05 & 0.000 \\
\hline Meat \& Preparations & & 1905.27 & 0.002 & & 2750.17 & 0.003 & & 3314.03 & 0.003 \\
\hline Marine Products & 483.52 & 6469.22 & 0.024 & 554.2 & 7035.91 & 0.020 & 611.55 & 8001.04 & 0.016 \\
\hline $\begin{array}{l}\text { Cotton Raw } \\
\text { including Waste }\end{array}$ & 86.64 & 422.58 & 0.000 & 614.8 & 2904.35 & 0.003 & 1162.22 & 6107.81 & 0.010 \\
\hline Jute Hessian & & 427.2 & 0.000 & & 490.9 & 0.000 & & 375.81 & 0.000 \\
\hline Poultry Products & & 281.96 & 0.000 & & 313.37 & 0.000 & & 313.82 & 0.000 \\
\hline $\begin{array}{l}\text { Paper/Wood } \\
\text { products }\end{array}$ & & 3236.66 & 0.006 & & 3759.15 & 0.006 & & 4915.22 & 0.006 \\
\hline $\begin{array}{l}\text { Total Agricultural } \\
\text { Exports }\end{array}$ & & 41602.65 & 1.000 & & 49216.96 & 1.000 & & 62411.42 & 1.000 \\
\hline $\begin{array}{l}\text { TotalNational } \\
\text { Exports }\end{array}$ & & 375339.53 & & & 456417.86 & & & 57177928 & \\
\hline$\%$ Share of & & 11.08 & & & 10.78 & & & 10.92 & \\
\hline $\begin{array}{l}\text { Agncultural Exports } \\
\text { in } \\
\text { National Exports }\end{array}$ & & & & & & & & & \\
\hline & & & 0.066 & & & 0.062 & & & 0.062 \\
\hline
\end{tabular}


Impact Of Global Economic Crisis On Export Diversification Of Agricultural Commodities: The Case Of Indian Horticulture Market

\begin{tabular}{|c|c|c|c|c|c|c|c|c|c|}
\hline \multirow{2}{*}{ Commodity } & \multicolumn{3}{|c|}{$2007-2008$} & \multicolumn{3}{|c|}{$2008-2009$} & \multicolumn{3}{|c|}{$2009-2010$} \\
\hline & Quantity & Value & $\begin{array}{r}\mathrm{HI} \\
(\mathrm{Ag})\end{array}$ & Quantity & Value & $\begin{array}{r}\mathrm{HI} \\
(\mathrm{Ag})\end{array}$ & Quantity & Value & $\begin{array}{r}\mathrm{HI} \\
(\mathrm{Ag})\end{array}$ \\
\hline Pulses & 164.20 & 526.41 & 0.000 & 136.27 & 540.22 & 0.000 & 100.00 & 407.36 & 0.000 \\
\hline Rice Basmati & 1183.36 & 4344.58 & 0.003 & 1556.41 & 9477.03 & 0.012 & 2015.91 & 10838.86 & 0.015 \\
\hline $\begin{array}{l}\text { Rice(Other than } \\
\text { Basmati) }\end{array}$ & 5286.08 & 7410.03 & 0.009 & 931.89 & 1687.37 & 0.000 & 139.37 & 414.76 & 0.000 \\
\hline Wheat & 0.24 & 0.24 & 0.000 & 1.12 & 1.46 & 0.000 & 0.00 & 0.00 & 0.000 \\
\hline Other Cereals & 3228.05 & 3002.33 & 0.001 & 3999.65 & 3920.58 & 0.002 & 2904.49 & 3004.93 & 0.001 \\
\hline Tea & 197.39 & 2034.17 & 0.001 & 207.46 & 2688.87 & 0.001 & 208.55 & 2943.27 & 0.001 \\
\hline Coffee & 178.30 & 1872.27 & 0.001 & 174.08 & 2255.76 & 0.001 & 177.23 & 2033.00 & 0.001 \\
\hline $\begin{array}{l}\text { Tobacco } \\
\text { Unmanufactured }\end{array}$ & 173.34 & 1432.80 & 0.000 & 208.31 & 2766.27 & 0.001 & 230.88 & 3621.24 & 0.002 \\
\hline $\begin{array}{l}\text { Tobacco } \\
\text { Manufactured }\end{array}$ & & 499.09 & 0.000 & & 294.78 & 0.000 & & 724.17 & 0.000 \\
\hline $\begin{array}{l}\text { Poultry \& Dairy } \\
\text { Products }\end{array}$ & & 960.24 & 0.000 & & 1130.08 & 0.000 & & 549.29 & 0.000 \\
\hline $\begin{array}{l}\text { Floriculture } \\
\text { Products }\end{array}$ & & 340.30 & 0.000 & & 368.81 & 0.000 & & 293.98 & 0.000 \\
\hline Spices & 614.86 & 4314.86 & 0.003 & 673.87 & 6338.42 & 0.005 & 680.60 & 6161.02 & 0.005 \\
\hline $\begin{array}{l}\text { Cashewnut Shell } \\
\text { Liquid }\end{array}$ & 14.78 & 25.17 & 0.000 & 10.82 & 29.69 & 0.000 & 11.23 & 27.62 & 0.000 \\
\hline Cashew & 111.26 & 2209.60 & 0.001 & 126.15 & 2900.97 & 0.001 & 122.17 & 2801.98 & 0.001 \\
\hline Sesamum Seed & 317.01 & 1642.29 & 0.000 & 196.98 & 1494.26 & 0.000 & 215.98 & 1495.38 & 0.000 \\
\hline Nigerseed & 21.68 & 90.23 & 0.000 & 13.72 & 64.23 & 0.000 & 6.00 & 24.23 & 0.000 \\
\hline Groundnut & 269.59 & 1054.08 & 0.000 & 297.89 & 1239.01 & 0.000 & 339.98 & 1424.55 & 0.000 \\
\hline Guargum Meal & 211.17 & 1125.75 & 0.000 & 258.57 & 1338.99 & 0.000 & 216.20 & 1132.87 & 0.000 \\
\hline Oil Meals & 6908.50 & 8140.55 & 0.011 & 6742.94 & 10269.24 & 0.014 & 4688.85 & 7849.57 & 0.008 \\
\hline Castor Oil & 282.18 & 1275.72 & 0.000 & 357.26 & 2128.72 & 0.001 & 397.70 & 2177.57 & 0.001 \\
\hline Shellac & 8.01 & 123.99 & 0.000 & 6.03 & 103.89 & 0.000 & 4.18 & 71.27 & 0.000 \\
\hline Sugar & 4684.55 & 5412.16 & 0.005 & 3332.08 & 4448.74 & 0.003 & 44.05 & 110.23 & 0.000 \\
\hline Molasses & 897.52 & 250.62 & 0.000 & 172.20 & 82.70 & 0.000 & 31.10 & 19.77 & 0.000 \\
\hline $\begin{array}{l}\text { Fruits/Vegetable } \\
\text { Seeds }\end{array}$ & 10.08 & 141.96 & 0.000 & 8.54 & 119.99 & 0.000 & 8.89 & 145.25 & 0.000 \\
\hline Fresh Fruits & & 1446.59 & 0.000 & & 1945.24 & 0.001 & & 2268.95 & 0.001 \\
\hline Fresh Vegetables & & 1477.89 & 0.000 & & 2454.15 & 0.001 & & 2904.35 & 0.001 \\
\hline $\begin{array}{l}\text { Processed } \\
\text { Vegetables }\end{array}$ & & 602.18 & 0.000 & & 711.22 & 0.000 & & 752.18 & 0.000 \\
\hline $\begin{array}{l}\text { Processed Fruit } \\
\text { Juices }\end{array}$ & & 773.40 & 0.000 & & 1099.15 & 0.000 & & 1155.95 & 0.000 \\
\hline \begin{tabular}{|l|} 
Miscellaneous \\
Processed Items
\end{tabular} & & 1362.39 & 0.000 & & 2077.44 & 0.001 & & 2136.85 & 0.001 \\
\hline Meat \& Preparations & & 3749.47 & 0.002 & & 5371.42 & 0.004 & & 6285.44 & 0.005 \\
\hline Marine Products & 490.06 & 6926.67 & 0.008 & 464.90 & 7066.37 & 0.007 & 720.23 & 9891.10 & 0.012 \\
\hline $\begin{array}{l}\text { Cotton Raw } \\
\text { including Waste }\end{array}$ & 1557.59 & 8865.39 & 0.013 & 457.56 & 2865.85 & 0.001 & 1360.27 & 9542.59 & 0.011 \\
\hline Jute Hessian & & 464.44 & 0.000 & & 415.59 & 0.000 & & 308.26 & 0.000 \\
\hline Poultry Products & & 429.53 & 0.000 & & 413.53 & 0.000 & & 365.85 & 0.000 \\
\hline $\begin{array}{l}\text { Paper/Wood } \\
\text { products }\end{array}$ & & 4712.33 & 0.004 & & 5441.63 & 0.004 & & 5638.90 & 0.004 \\
\hline $\begin{array}{l}\text { Total Agricultural } \\
\text { Exports }\end{array}$ & & 79039.72 & 1.000 & & 85951.67 & 1.000 & & 89522.59 & 1.000 \\
\hline $\begin{array}{l}\text { TotalNational } \\
\text { Exports }\end{array}$ & & 655863.52 & & & 840755.06 & & & 845125.21 & \\
\hline \multirow{3}{*}{$\begin{array}{l}\text { \% Share of } \\
\text { Agricultural Exports } \\
\text { in } \\
\text { National Exports }\end{array}$} & & 12.05 & & & 10.22 & & & 10.59 & \\
\hline & & & & & & & & & \\
\hline & & & 0.062 & & & 0.060 & & & 0.069 \\
\hline
\end{tabular}


Annexure: 2

India's Export of selected Horticultural products (Fruits)

(Value: In Rs. Crores)

\begin{tabular}{|c|c|c|c|c|}
\hline Commodity & 2001-2002 & HI (Ag) & $2002-2003$ & HI (Ag) \\
\hline BANANAS & $1,583.94$ & $4.06167 \mathrm{E}-05$ & $1,272.37$ & $1.99515 \mathrm{E}-05$ \\
\hline PINEAPPLES & 159.97 & $4.1429 \mathrm{E}-07$ & 142.23 & $2.49305 \mathrm{E}-07$ \\
\hline ORANGES & $3,187.54$ & 0.00016449 & $2,846.72$ & $9.98708 \mathrm{E}-05$ \\
\hline GUAVAS & 172.3 & $4.80616 \mathrm{E}-07$ & 207.44 & $5.30315 \mathrm{E}-07$ \\
\hline LICHI & 35.99 & $2.09697 \mathrm{E}-08$ & 100.22 & $1.23782 \mathrm{E}-07$ \\
\hline MANGO & $33,252.73$ & 0.017901202 & $39,104.00$ & 0.018844789 \\
\hline APPLE & $1,339.53$ & $2.90491 \mathrm{E}-05$ & $1,572.72$ & $3.04826 \mathrm{E}-05$ \\
\hline GRAPES & $6,048.81$ & 0.000592335 & $11,029.58$ & 0.001499225 \\
\hline POMEGRANATES & $1,041.85$ & $1.75727 \mathrm{E}-05$ & $1,434.57$ & $2.53625 \mathrm{E}-05$ \\
\hline PAPAYAS & 286.89 & $1.33247 \mathrm{E}-06$ & 475.92 & $2.79137 \mathrm{E}-06$ \\
\hline WATER MELONS & 248.40 & 9.9892E-07 & 276.00 & $9.38787 \mathrm{E}-07$ \\
\hline $\begin{array}{l}\text { TOTAL (EDIBLE } \\
\text { FRUITS AND } \\
\text { NUTS - ALL } \\
\text { FORMS) }\end{array}$ & $248,534.23$ & 1 & $284,856.11$ & 1 \\
\hline $\begin{array}{l}\text { INDIA'S TOTAL } \\
\text { EXPORTS } \\
\end{array}$ & 20,901,797.34 & & $25,513,727.66$ & \\
\hline
\end{tabular}

\begin{tabular}{|l|r|c|r|r|}
\hline \multicolumn{1}{|c|}{ Commodity } & \multicolumn{1}{|c|}{$\mathbf{2 0 0 3 - 2 0 0 4}$} & \multicolumn{1}{c|}{ HI (Ag) } & \multicolumn{1}{c|}{$\mathbf{2 0 0 4 - 2 0 0 5}$} & \multicolumn{1}{c|}{ HI (Ag) } \\
\hline BANANAS & $1,171.82$ & $2.11724 \mathrm{E}-05$ & $1,342.76$ & $1.55603 \mathrm{E}-05$ \\
\hline PINEAPPLES & 201.71 & $6.27341 \mathrm{E}-07$ & 245.15 & $5.18662 \mathrm{E}-07$ \\
\hline ORANGES & $5,227.59$ & 0.000421358 & $3,300.66$ & $9.40205 \mathrm{E}-05$ \\
\hline GUAVAS & 568.01 & $4.97463 \mathrm{E}-06$ & 692.62 & $4.1401 \mathrm{E}-06$ \\
\hline LICHI & 133.98 & $2.76776 \mathrm{E}-07$ & 70.9 & $4.33824 \mathrm{E}-08$ \\
\hline MANGO & $39,102.00$ & 0.023574727 & $43,971.04$ & 0.01668608 \\
\hline APPLE & $1,320.08$ & $2.68689 \mathrm{E}-05$ & $2,644.49$ & $6.03538 \mathrm{E}-05$ \\
\hline GRAPES & $10,603.66$ & 0.001733646 & $12,883.64$ & 0.001432511 \\
\hline POMEGRANATES & $2,109.00$ & $6.85807 \mathrm{E}-05$ & $2,988.71$ & $7.70883 \mathrm{E}-05$ \\
\hline PAPAYAS & 460.56 & $3.27055 \mathrm{E}-06$ & 531.2 & $2.43521 \mathrm{E}-06$ \\
\hline WATER MELONS & 223.66 & $7.71304 \mathrm{E}-07$ & 386.52 & $1.28933 \mathrm{E}-06$ \\
\hline $\begin{array}{l}\text { TOTAL (EDIBLE } \\
\text { FRUITS AND NUTS } \\
\text { - ALL FORMS) }\end{array}$ & $\mathbf{2 5 4 , 6 6 8 . 7 4}$ & & $\mathbf{3 4 0 , 4 0 0 . 0 2}$ & \\
\hline $\begin{array}{l}\text { INDIA'S TOTAL } \\
\text { EXPORTS }\end{array}$ & & & & 1 \\
\hline & $\mathbf{2 9 , 3 3 6 , 6 7 4 . 7 5}$ & & $\mathbf{3 7 , 5 3 3 , 9 5 2 . 6 2}$ & \\
\hline
\end{tabular}




\begin{tabular}{|c|c|c|c|c|}
\hline Commodity & $2005-2006$ & HI (Ag) & 2006-2007 & HI (Ag) \\
\hline BANANAS & $2,336.19$ & $3.8535 \mathrm{E}-05$ & $1,606.78$ & $1.62809 \mathrm{E}-05$ \\
\hline PINEAPPLES & 515.49 & 1.8762E-06 & 360.96 & $8.21643 \mathrm{E}-07$ \\
\hline ORANGES & $3,487.25$ & $8.5862 \mathrm{E}-05$ & $2,913.86$ & $5.3543 \mathrm{E}-05$ \\
\hline GUAVAS & 982.98 & $6.8222 \mathrm{E}-06$ & 772.53 & $3.76353 \mathrm{E}-06$ \\
\hline LICHI & 93.64 & $6.191 \mathrm{E}-08$ & 155.94 & $1.53349 \mathrm{E}-07$ \\
\hline MANGO & $51,437.55$ & 0.01868082 & $67,936.33$ & 0.029105117 \\
\hline APPLE & $3,892.80$ & 0.00010699 & $3,151.43$ & $6.26297 \mathrm{E}-05$ \\
\hline GRAPES & $21,607.20$ & 0.00329634 & $30,527.48$ & 0.005876884 \\
\hline POMEGRANATES & $5,670.16$ & 0.000227 & $11,461.62$ & 0.000828433 \\
\hline PAPAYAS & 665.13 & $3.1236 \mathrm{E}-06$ & $1,160.11$ & $8.48718 \mathrm{E}-06$ \\
\hline WATER MELONS & 507.17 & $1.8161 \mathrm{E}-06$ & 750.52 & $3.55214 \mathrm{E}-06$ \\
\hline $\begin{array}{l}\text { TOTAL (EDIBLE } \\
\text { FRUITS AND NUTS - } \\
\text { ALL FORMS) }\end{array}$ & $376,341.66$ & 1 & $398,214.81$ & \\
\hline $\begin{array}{l}\text { INDIA'S TOTAL } \\
\text { EXPORTS }\end{array}$ & $45,641,786.15$ & & $57,177,928.52$ & \\
\hline & & 0.02244925 & & 0.03595966 \\
\hline Commodity & $2007-2008$ & HI (Ag) & 2008-2009 & HI (Ag) \\
\hline BANANAS & $2,607.95$ & $4.86368 \mathrm{E}-05$ & $5,545.76$ & 0.000125283 \\
\hline PINEAPPLES & 339.76 & $8.25488 \mathrm{E}-07$ & 367.81 & $5.51084 \mathrm{E}-07$ \\
\hline ORANGES & $2,726.83$ & $5.31719 \mathrm{E}-05$ & $2,320.34$ & $2.19318 \mathrm{E}-05$ \\
\hline GUAVAS & 417.19 & $1.24461 \mathrm{E}-06$ & 304.42 & $3.775 \mathrm{E}-07$ \\
\hline LICHI & 88.91 & $5.65285 \mathrm{E}-08$ & 155.94 & $9.90571 \mathrm{E}-08$ \\
\hline MANGO & $65,914.45$ & 0.031069031 & $93,097.96$ & 0.035306179 \\
\hline APPLE & $3,335.20$ & $7.95445 \mathrm{E}-05$ & $5,232.39$ & 0.000111525 \\
\hline GRAPES & $31,706.78$ & 0.007189037 & $37,465.10$ & 0.005717737 \\
\hline POMEGRANATES & $11,942.84$ & 0.001019957 & $11,461.62$ & 0.000535134 \\
\hline PAPAYAS & $1,123.18$ & $9.02122 \mathrm{E}-06$ & $1,809.58$ & $1.33391 \mathrm{E}-05$ \\
\hline WATER MELONS & 579.69 & $2.40302 \mathrm{E}-06$ & $1,048.79$ & $4.48072 \mathrm{E}-06$ \\
\hline $\begin{array}{l}\text { TOTAL (EDIBLE } \\
\text { FRUITS AND } \\
\text { NUTS - ALL } \\
\text { FORMS) } \\
\end{array}$ & $373,952.79$ & 1 & $495,467.08$ & 1 \\
\hline $\begin{array}{l}\text { INDIA'S TOTAL } \\
\text { EXPORTS }\end{array}$ & $65,586,352.18$ & & $84,075,505.87$ & \\
\hline & & 0.03947293 & & 0.04183664 \\
\hline
\end{tabular}




\begin{tabular}{|l|l|l|}
\hline Commodity & $\mathbf{2 0 0 9 - 2 0 1 0}$ & HI (Ag) \\
\hline BANANAS & $13,014.65$ & 0.000628132 \\
\hline PINEAPPLES & 402.7 & $6.0138 \mathrm{E}-07$ \\
\hline ORANGES & $2,539.26$ & $2.39111 \mathrm{E}-05$ \\
\hline GUAVAS & 113.39 & $4.76799 \mathrm{E}-08$ \\
\hline LICHI & 88.91 & $2.93148 \mathrm{E}-08$ \\
\hline MANGO & $95,582.44$ & 0.033879884 \\
\hline APPLE & $3,210.34$ & $3.82197 \mathrm{E}-05$ \\
\hline GRAPES & $43,832.07$ & 0.00712475 \\
\hline POMEGRANATES & $11,942.84$ & 0.000528933 \\
\hline PAPAYAS & $1,748.75$ & $1.13407 \mathrm{E}-05$ \\
\hline WATER MELONS & $1,665.64$ & $1.02884 \mathrm{E}-05$ \\
\hline $\begin{array}{l}\text { TOTAL (EDIBLE FRUITS AND NUTS - ALL } \\
\text { FORMS) }\end{array}$ & $\mathbf{5 1 9 , 2 8 6 . 6 5}$ & 1 \\
\hline INDIA'S TOTAL EXPORTS & $\mathbf{8 4 , 5 5 3 , 3 6 4 . 3 8}$ & \\
\hline & & $\mathbf{0 . 0 4 2 2 4 6 1}$ \\
\hline
\end{tabular}

Annexure: 3

India's Export of selected Horticultural products (Vegetables)

(Value: In Rs. Crores)

\begin{tabular}{|l|r|r|r|c|}
\hline \multicolumn{1}{|c|}{ Commodity } & $\mathbf{2 0 0 1 - 2 0 0 2}$ & \multicolumn{1}{c|}{ HI (Ag) } & \multicolumn{1}{c|}{$\mathbf{2 0 0 2 - 2 0 0 3}$} & HI (Ag) \\
\hline POTATOES & 744.47 & $4.37818 \mathrm{E}-05$ & 1747.97 & 0.00020279 \\
\hline TOMATOES & 136.47 & $1.47121 \mathrm{E}-06$ & $1,185.05$ & $9.3209 \mathrm{E}-05$ \\
\hline ONIONS & 39003.41 & 0.12017228 & $44,471.83$ & 0.13126731 \\
\hline GARLIC & 177.8 & $2.49725 \mathrm{E}-06$ & 698.67 & $3.2399 \mathrm{E}-05$ \\
\hline $\begin{array}{l}\text { CARROTS \& } \\
\text { TURNIPS }\end{array}$ & 2.24 & $3.96365 \mathrm{E}-10$ & 3.73 & $9.2343 \mathrm{E}-10$ \\
\hline PEAS & 307.28 & $7.45878 \mathrm{E}-06$ & 1311.59 & 0.00011418 \\
\hline MUSHROOM & 7836.56 & 0.00485121 & 6687.59 & 0.00296842 \\
\hline GREEN CHILLY & 285.95 & $6.45921 \mathrm{E}-06$ & 284.47 & $5.3711 \mathrm{E}-06$ \\
\hline SPINACH & 0.52 & $2.13602 \mathrm{E}-11$ & 0.27 & $4.8385 \mathrm{E}-12$ \\
\hline PUMPKINS & 0.92 & $6.68613 \mathrm{E}-11$ & 6.46 & $2.7698 \mathrm{E}-09$ \\
\hline CHICKPEAS & 254.06 & $5.09884 \mathrm{E}-06$ & 402.34 & $1.0744 \mathrm{E}-05$ \\
\hline $\begin{array}{l}\text { TOTAL EDIBLE } \\
\text { VEGETABLES, } \\
\text { ROOTS AND } \\
\text { TUBERS (ALL } \\
\text { FORMS) }\end{array}$ & $\mathbf{1 1 2 , 5 1 2 . 4 1}$ & & $\mathbf{1 2 2 , 7 4 5 . 8 2}$ & \\
\hline $\begin{array}{l}\text { INDIA'S TOTAL } \\
\text { EXPORTS }\end{array}$ & & & & \\
\hline & & & & 1 \\
\hline
\end{tabular}




\begin{tabular}{|l|r|r|r|r|}
\hline \multicolumn{1}{|c|}{ Commodity } & $\mathbf{2 0 0 3 - 2 0 0 4}$ & \multicolumn{1}{c|}{ HI (Ag) } & \multicolumn{1}{c|}{$\mathbf{2 0 0 4 - 2 0 0 5}$} & \multicolumn{1}{c|}{ HI (Ag) } \\
\hline POTATOES & 3172.73 & 0.00044147 & 3392.14 & 0.000386884 \\
\hline TOMATOES & 685.42 & $2.0604 \mathrm{E}-05$ & 589.38 & $1.16795 \mathrm{E}-05$ \\
\hline ONIONS & 77009.46 & 0.26009225 & 69099.84 & 0.16054164 \\
\hline GARLIC & 1422.65 & $8.8764 \mathrm{E}-05$ & 438.19 & $6.45593 \mathrm{E}-06$ \\
\hline $\begin{array}{l}\text { CARROTS \& } \\
\text { TURNIPS }\end{array}$ & 13.18 & & 11.24 & $4.24782 \mathrm{E}-09$ \\
\hline PEAS & 1423.92 & $8.8922 \mathrm{E}-05$ & 1695.05 & $9.66048 \mathrm{E}-05$ \\
\hline MUSHROOM & 4734.26 & 0.00098298 & 3443.87 & 0.000398774 \\
\hline GREEN CHILLY & 898.99 & $3.5445 \mathrm{E}-05$ & 909.21 & $2.77947 \mathrm{E}-05$ \\
\hline SPINACH & 3.64 & $5.8109 \mathrm{E}-10$ & 28.16 & $2.66624 \mathrm{E}-08$ \\
\hline PUMPKINS & 0.0008 & $2.8069 \mathrm{E}-17$ & 318.24 & $3.4052 \mathrm{E}-06$ \\
\hline CHICKPEAS & $\mathbf{5 3 9 . 8 8}$ & $1.2783 \mathrm{E}-05$ & $4,127.76$ & 0.000572878 \\
\hline $\begin{array}{l}\text { TOTAL EDIBLE } \\
\text { VEGETABLES, } \\
\text { ROOTS AND } \\
\text { TUBERS (ALL } \\
\text { FORMS) }\end{array}$ & $\mathbf{1 5 1 , 0 0 1 . 1 9}$ & & $\mathbf{1 7 2 , 4 5 7 . 9 4}$ & \\
\hline $\begin{array}{l}\text { INDIA'S TOTAL } \\
\text { EXPORTS }\end{array}$ & $\mathbf{2 9 , 3 3 6 , 6 7 4 . 7 5}$ & & & \\
\hline & & $\mathbf{0 . 2 6 1 7 6 3 2}$ & & \\
\hline
\end{tabular}

\begin{tabular}{|c|c|c|c|c|}
\hline Commodity & $2005-2006$ & HI (Ag) & 2006-2007 & HI (Ag) \\
\hline POTATOES & 4683.85 & 0.00034707 & 6696.56 & 0.00056343 \\
\hline TOMATOES & $1,086.07$ & $1.8661 \mathrm{E}-05$ & $3,410.20$ & 0.00014611 \\
\hline ONIONS & 81383.67 & 0.1047831 & 132065.86 & 0.21913663 \\
\hline GARLIC & 3875.61 & 0.00023763 & 1996.35 & $5.0073 \mathrm{E}-05$ \\
\hline CARROTS \& TURNIPS & 19.03 & 5.7292E-09 & 19.32 & 4.6897E-09 \\
\hline PEAS & 1975.31 & $6.1729 \mathrm{E}-05$ & 917.15 & $1.0569 \mathrm{E}-05$ \\
\hline MUSHROOM & 7042.9 & 0.00078473 & 3967.45 & 0.00019777 \\
\hline GREEN CHILLY & $1,482.59$ & $3.4774 \mathrm{E}-05$ & $2,216.38$ & 6.1719E-05 \\
\hline SPINACH & 81.81 & $1.0588 \mathrm{E}-07$ & 40.83 & $2.0946 \mathrm{E}-08$ \\
\hline PUMPKINS & 662.25 & $6.9384 \mathrm{E}-06$ & 635.94 & $5.0812 \mathrm{E}-06$ \\
\hline CHICKPEAS & $15,069.64$ & 0.00359271 & $21,730.51$ & 0.00593299 \\
\hline $\begin{array}{l}\text { TOTAL EDIBLE } \\
\text { VEGETABLES, } \\
\text { ROOTS AND } \\
\text { TUBERS (ALL } \\
\text { FORMS) }\end{array}$ & $251,415.27$ & 1 & $282,119.48$ & . \\
\hline \multirow[t]{2}{*}{$\begin{array}{l}\text { INDIA'S TOTAL } \\
\text { EXPORTS }\end{array}$} & $45,641,786.15$ & & $57,177,928.52$ & \\
\hline & & 0.1098675 & & 0.2261044 \\
\hline
\end{tabular}




\begin{tabular}{|l|r|r|r|r|}
\hline \multicolumn{1}{|c|}{ Commodity } & \multicolumn{1}{c|}{$\mathbf{2 0 0 7 - 2 0 0 8}$} & \multicolumn{1}{c|}{ HI (Ag) } & \multicolumn{1}{c|}{$\mathbf{2 0 0 8 - 2 0 0 9}$} & \multicolumn{1}{c|}{ HI (Ag) } \\
\hline POTATOES & 4867.16 & 0.000372332 & 13810.41 & 0.000417559 \\
\hline TOMATOES & $15,290.78$ & 0.003674838 & $12,766.20$ & 0.000661495 \\
\hline ONIONS & 126618.91 & 0.251985826 & $197,069.35$ & 0.406102089 \\
\hline GARLIC & 170.64 & $4.57658 \mathrm{E}-07$ & 242.18 & 0.00012617 \\
\hline $\begin{array}{l}\text { CARROTS \& } \\
\text { TURNIPS }\end{array}$ & 38.23 & $2.29714 \mathrm{E}-08$ & 67.65 & $2.90433 \mathrm{E}-08$ \\
\hline PEAS & 929.51 & $1.35796 \mathrm{E}-05$ & 2052.81 & $1.22644 \mathrm{E}-05$ \\
\hline MUSHROOM & 3084.13 & 0.000149501 & 1681.56 & $3.03669 \mathrm{E}-05$ \\
\hline GREEN CHILLY & $4,372.16$ & 0.000300449 & $4,832.06$ & 0.000245303 \\
\hline SPINACH & 66.63 & $6.9778 \mathrm{E}-08$ & 89.09 & $1.97128 \mathrm{E}-08$ \\
\hline PUMPKINS & $2,099.36$ & $6.92712 \mathrm{E}-05$ & 365.69 & $1.57841 \mathrm{E}-06$ \\
\hline CHICKPEAS & $51,896.93$ & 0.042331368 & $50,276.19$ & 0.009491178 \\
\hline $\begin{array}{l}\text { TOTAL EDIBLE } \\
\text { VEGETABLES, } \\
\text { ROOTS AND } \\
\text { TUBERS (ALL } \\
\text { FORMS) }\end{array}$ & $\mathbf{2 5 2 , 2 3 8 . 0 0}$ & & $\mathbf{3 5 6 , 2 4 1 . 2 0}$ & \\
\hline $\begin{array}{l}\text { INDIA'S TOTAL } \\
\text { EXPORTS }\end{array}$ & & & & 1 \\
\hline & & & & \\
\hline
\end{tabular}

\begin{tabular}{|l|r|r|}
\hline \multicolumn{1}{|c|}{ Commodity } & \multicolumn{1}{c|}{$\mathbf{2 0 0 9 - 2 0 1 0}$} & \multicolumn{1}{c|}{ HI (Ag) } \\
\hline POTATOES & 8202.67 & 0.00041756 \\
\hline TOMATOES & $10,324.28$ & 0.00066149 \\
\hline ONIONS & 255808.03 & 0.40610209 \\
\hline GARLIC & 4508.95 & 0.00012617 \\
\hline CARROTS \& TURNIPS & 68.41 & $2.9043 \mathrm{E}-08$ \\
\hline PEAS & 1405.79 & $1.2264 \mathrm{E}-05$ \\
\hline MUSHROOM & 2212.06 & $3.0367 \mathrm{E}-05$ \\
\hline GREEN CHILLY & $6,287.07$ & 0.0002453 \\
\hline SPINACH & 56.36 & $1.9713 \mathrm{E}-08$ \\
\hline PUMPKINS & 504.32 & $1.5784 \mathrm{E}-06$ \\
\hline CHICKPEAS & $39,107.19$ & 0.00949118 \\
\hline $\begin{array}{l}\text { TOTAL EDIBLE VEGETABLES, } \\
\text { ROOTS AND TUBERS (ALL }\end{array}$ & $\mathbf{4 0 1 , 4 1 7 . 7 4}$ & \\
FORMS) & & \\
\hline INDIA'S TOTAL EXPORTS & $\mathbf{8 4 , 5 5 3 , 3 6 4 . 3 8}$ & \\
\hline \multicolumn{2}{|c|}{} & \\
\hline
\end{tabular}

